\title{
PERFORMANCE OF A 2048 X 2048 PIXEL THREE-SIDE-BUTTABLE CCD DESIGNED FOR LARGE FOCAL PLANES IN ASTRONOMY
}

\author{
J. A. Cortiula \\ Thomson-CSF Semiconducteurs Specifiques
}

\begin{abstract}
TH78997M is a $2048 \times 2048$ pixel full-frame CCD sensor featuring $15 \times 15$ micron pixels, MPP operating mode, four parallel outputs and buttability on three sides. This new device makes it possible to build very large focal plane detectors for telescopes (two x $\mathrm{n}$ butted CCDs) with less than a 25 dead pixel zone between adjacent sensors. Other features are high full-well capacity (typically $180 \mathrm{Ke}^{-}$) and very low dark current thanks to four phase MPP clocking which allows a very long exposure time together with a high $\mathrm{S} / \mathrm{N}$ ratio.

The readout of a complete frame is achieved through four parallel outputs running at frequencies ranging from ten $\mathrm{KHz}$ up to five $\mathrm{MHz}$ per output. The four on-chip amplifiers have been designed to be completely user controlled and to meet most of terrestrial and spaceborne astronomy requirements: very low noise (less than $4 \mathrm{e}^{-}$at $50 \mathrm{KHz},-40$ degrees $\mathrm{C}$ ), very low power consumption ( $150 \mathrm{micro}-\mathrm{W} /$ amplifier at $10 \mathrm{KHz} /$ output), linearity better than $0.5 \%$ together with high conversion factor $\left(4.2 \mathrm{micro}-\mathrm{V} / \mathrm{e}^{-}\right)$over $2.5 \mathrm{~V}$ output range. The packaging of the device is compatible with the good flatness of the chip and with the low parasitic "cosmic event" rate thanks to specific care in selecting the packaging material.
\end{abstract}




\section{DISCUSSION}

IWERT: To avoid possible confusion, I would like to point out that the presented frontside product, THX 7837M, differs considerably from the product, THX 7397M, under development by Thomson for ESO. The frontside product, THX 7837M, has four outputs. They are arranged that way because the operation of all outputs is needed to read out the full CCD imaging area. The backside product, THX 7397M, is an independent development for ESO, taking thinning into account right from the start. This device has two outputs in the conventional scheme. There are many other differences between those two product developments, which cannot be covered in this context. However the fabrication process technology used for those two products is the same and the results on cosmetics and amplifier similarity due to a clean process are certainly encouraging. 